\title{
CORRESPONDENCE
}

Replies to 'The evolution of eusociality’ by Nowaket al. (Nature 466, 1057-1062; 2010). SEE COMMENT P.653

\section{Better living through physics}

Nowak and colleagues' explanation of the evolution of altruism in terms of individuallevel selection might be reconciled with the views of their kin-selection opponents by striking an analogy with statistical mechanical and thermodynamic treatments in physics.

Statistical mechanics provides the microscopic basis for the macroscopic variables in thermodynamics, which is an equilibrium theory treating aggregate variables. As with thermodynamics, traditional multilevel selection theory is based on equilibrium solutions operating on nominal, aggregate variables. In the Hamilton kinselection framework, variables correspond to the terms benefit, cost and relatedness. But because that treatment is not fundamentally mechanistic, it is often unclear what the units of these variables are, and how best to measure them.

Population genetics presents an evolutionary analogue of statistical mechanics that complements Hamilton's evolutionary thermodynamics. Hamilton's rule - which expresses relatedness between the helped and the helper in terms of cost and benefit to the fitness of both - and its related inequalities all express dependencies among macroscopic variables of state in structured populations.

The greater complexity of biological systems over physical ones, and their strong interdependency, make for a zoo of biological macroscopic laws with many multilevel selection principles, each with its adherents and disciples.

The great promise of evolutionary statistical mechanics is that it should allow us to enumerate the full space of possible fundamental evolutionary inequalities and the mechanistic conditions under which they apply, thence identifying those with the greatest empirical generality.

David C. Krakauer, Jessica C. Flack Santa Fe Institute, USA, krakauer@santafe.edu

\section{Inclusive fitness is just bookkeeping}

Using a very general modelling approach, Nowak and colleagues make two important and valid points. First, when standard natural selection theory and inclusive fitness theory can both be applied, they must yield the same answer. Whether the realm of applicability of inclusive fitness models is as restricted as claimed remains to be seen, but it is in any case contained in the realm of standard models. Second, the paper makes it clear what inclusive fitness theory really is: an accounting method, not a biological mechanism.

Champions of inclusive fitness often refer to the underlying mechanism as kin selection, but this just restates the fact that the benefit a particular gene generates at a cost to its carrier must preferentially go to the gene's other carriers (kin). The real biological problem is to understand mechanisms that lead to such assortment between helper and help. For eusocial insects, Nowak et al. convincingly argue that the basic mechanism of assortment is the formation of groups owing to ecological pressures, such as the need for nest defence.

Despite the indignant response of the inclusive-fitness crowd, there can be no doubt about the fundamental tenet that, with or without the concept of inclusive fitness, in principle we have access to exactly the same amount of evolutionary knowledge. Personal modelling preferences may vary, but there is nothing magic about bookkeeping techniques.

Michael Doebeli University of British Columbia, Canada, doebeli@zoology.ubc.ca

\section{Ground truth is the test that counts}

A strength of kin-selection theory for explaining the evolution of altruism is that it generates multiple hypotheses that can fail in resulting tests, forcing principles to be re-examined. Lessons can be learned from one such failure discussed by Nowak et al:: the haplodiploid hypothesis.

With haplodiploidy, fertilized eggs become females and unfertilized ones become males. Thus, females can have higher fitness if they raise their full sisters instead of their own offspring.

This prediction was not wrong in the sense that it arose from flawed mathematics. Indeed, practitioners of the naturalselection approach championed by Nowak et al. could have made the exact same prediction. It fails experimentally because a basic assumption is not met in nature: females rarely get the opportunity preferentially to raise full sisters.

Theoretical papers that developed more viable alternative scenarios also used inclusive fitness methods (for example, see D. C. Queller Proc. Natl Acad. Sci. USA 86, 3224-3226; 1989). This robust predict-fail-reevaluate triumvirate is why kin selection has been the main informative model of choice in the past and why it should continue to help us understand the evolution of cooperation and conflict. Peter Nonacs University of California, Los Angeles, USA, pnonacs@biology.ucla.edu

\section{Call for a return to rigour in models}

Why are numerous reactions to the Nowak et al. paper so ferocious? And how is it possible that theorists even seem to disagree about mathematics? An important key to the heatedness of the debate is that many theory papers on the evolution of cooperation use the Price equation. This is regularly treated as if its generality makes it the $E=m c^{2}$ of population genetics. Those who use it, however, tend to forget that it is only general because it contains no modelling assumptions. And without these, one cannot derive theoretical predictions.

The Price equation inspires theoreticians to confuse identity with causality, and probability theory with statistics (M. van Veelen J. Theor. Biol. 237, 412$426 ; 2005)$. It is this theoretical blur that obfuscates whether or not claims are theoretical results that follow from actual model assumptions.

References to 'results' derived with the Price equation are a recurring element in discussions of inclusive fitness. As long as the Price equation is thought useful for finding theoretical predictions, these 'results' will collide with results from actual models, which is a recipe for hot-headed debates.

Nowak and colleagues' paper is exciting because it goes back to basics: it builds proper models. Rather than saying the paper is wrong, it would be more fruitful if critics also went back to basics: state model assumptions, derive predictions, test empirically. Such a return to rigour would help the field advance to the next level. Matthijs van Veelen, Julián García, Maurice W. Sabelis, Martijn Egas University of Amsterdam, the Netherlands, c.m.vanveelen@uva.nl 